\title{
Impact of health marketing on the intake of sugars by children
}

\author{
G. Gardner ${ }^{1,2}$, P. Moynihan ${ }^{2}$, E. Foster ${ }^{1}$, R. Holmes ${ }^{2}$, A. Don Bosco ${ }^{3}$ and O. Hugeniot ${ }^{3}$ \\ ${ }^{1}$ Institute for Health and Society, Newcastle University, NE2 $4 A X, U K,{ }^{2}$ Centre for Oral Health Research, \\ Newcastle University, NE2 4BW, UK and ${ }^{3}$ Public Health England, EC4Y 8JX, UK.
}

The UK population consumes too much Free Sugars, which is also the case in other countries - making it a problem of global importance $^{(1,2)}$. The negative consequences of Free Sugars intake include dental caries and obesity, and effective interventions to reduce this intake are therefore needed. Public Health England launched a Change4Life 'Sugar Smart' campaign in 2016, as part of their range of measures aiming to combat childhood obesity. The aims of this project were 1) to determine any impact of the Sugar Smart Campaign on dietary behaviour and 2) to evaluate the impact of the campaign on parental awareness of dietary sugars, and parental perception of any impact on their child's sugars intake.

A mixed methods evaluation was used to examine the impact of the campaign on the intake of sugars by boys and girls aged 5-11 years, from a range of socioeconomic backgrounds from across England. The social marketing company TNS BMRB was used to recruit participants who had previously signed up to Change4Life campaigns. Participants were asked to complete two dietary recalls at 5-time points; pre-campaign, peak-campaign and 1, 10 and 12-months post-campaign using the online dietary recall tool INTAKE24 ${ }^{(3)}$. Any changes in Total and Free Sugars intake was determined using paired analysis.

A purposive sub-sample of 44 parents who had previously completed dietary recalls were contacted by email one-year postcampaign and asked to take part in an additional one-to-one semi-structured telephone interview. Interviews were digitally recorded, transcribed verbatim, and thematically coded using NVivo 11.0 software. The constant comparative technique was employed, along with the Framework analysis approach.

Dietary recalls were completed by 539 parents. A statistically significant decrease was reported in the percentage energy from Total Sugars across all time points compared to baseline, including 12 -months post campaign $(0.9 \%$, p < 0.05). Percentage energy from non-milk extrinsic sugars significantly decreased at peak campaign $(0.9 \%, \mathrm{p}<0.05)$, however, there was no significant decrease at any post campaign time point. A decrease in the contribution of fresh fruit to total sugars intake over the 12-month period was also found.

Data saturation was reached at 27 qualitative interviews. Analysis identified an overall raised parental awareness of sugars intake by their children, as well as a raised knowledge of sugars in general, one-year post campaign. There was a parental desire to reduce child sugars intake, and the campaign facilitated changes in some household shopping such as a reduction in the purchasing of sugary cereals and drinks. However, a number of barriers to reducing sugars intake were reported including cost, hidden sugars and habit.

The national Sugar Smart health marketing campaign had a positive short-term impact in reducing Free Sugars intake by children. Qualitative findings suggest the approach was successful in driving the desire for change, as the campaign helped to facilitate a culture change, an improved consumer demand, and behaviour change. Parents perceived the campaign to be successful in providing them with a raised awareness and understanding of sugars intake by their children, one-year following the campaign.

1. Public Health England. National Diet and Nutrition Survey. Results from Years 5 and 6 (combined) of the Rolling Programme (2012/13-2013/14). https://www.gov.uk/government/statistics/ndns-results-from-years-5-and-6-combined

2. The Scientific Advisory Committee on Nutrition. (2015) Carbohydrates and Health. https://www.gov.uk/government/groups/scientific-advisorycommittee-on-nutrition

3. Simpson E, Bradley J, Poliakov I, Jackson D, Oliver P, Adamson AJ \& Foster E. (2017) Iterative development of an online dietary recall tool: INTAKE24. Nutrients, $9(2)$. 